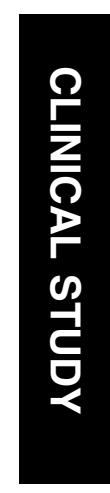

${ }^{1}$ Department of

Ophthalmology, School of Medicine, Düzce University, Düzce, Turkey

${ }^{2}$ Beyoglu Eye Training and Research Hospital, Second Eye Clinic, Istanbul, Turkey

${ }^{3}$ Department of Biostatistics, School of Medicine, Marmara University, Istanbul, Turkey

${ }^{4}$ Interactive Multi-Media Technology Center, Georgia Institute of Technology, Atlanta, GA, USA

${ }^{5}$ Vimetrics, LLC, Media, Philadelphia, PA, USA

${ }^{6}$ Department of Ophthalmology, School of Medicine, Drexel University, Philadelphia, PA, USA

Correspondence: SH Sinclair, Department of Ophthalmology, School of Medicine, Drexel University, 311 East Baltimore Ave, 1st floor, Suite 100, Media, PA 19063, USA Tel: + 6108921708; Fax: +6108928766

E-mail: stephensinclair@ mac.com

Received: 17 November 2008

Accepted in revised form: 11 June 2009 Published online: 17 July 2009

Financial disclosure: Stephen $\mathrm{H}$. Sinclair and Peter Presti have financial interests in Vimetrics, LLC, which intends to market the device.

\title{
Central field perimetry of discriminated targets: I. Results for normal individuals using high-contrast targets
}

\begin{abstract}
Purpose A new perimetry method is described to evaluate central vision under multiple hue, illumination, and contrast conditions; results are described for normal individuals using high-contrast targets. Methods For 70 normal eyes of individuals with varying age, the ETDRS chart acuity was determined as well as discriminated target perimetry using high-contrast targets presented for $250 \mathrm{~ms}$ at locations up to $10^{\circ}$ eccentricity. Retesting was performed to evaluate for learning and repeatability for the thresholded acuity at each eccentricity. Results The ETDRS acuity averaged $1.15 \pm 0.37$ arc min minimum angle of
\end{abstract} resolution (MAR) with best correction (20/23 equivalence). At fixation, the thresholded acuity for high-contrast targets paralleled the ETDRS acuity and averaged $1.75 \pm 0.85$ arc min MAR (20/35 equivalence), and declined with increasing eccentricity in a linear fashion to $5.81 \pm 3.97$ arc $\min (20 / 116$ equivalent) at $10^{\circ}$ eccentricity. Linear regression showed Vmar $=1.74+0.330 *$ eccentricity in degrees $\left(R^{2}=0.966, P<0.0001\right)$. Testing time required $\sim$ 8-10 min per eye. Repeated testing showed only minimal learning experience at the most peripheral locations. The test-retest $\mathbf{9 5 \%}$ confidence limits of difference measured 1.12 arc mins MAR at fixation $(64 \%$ of the mean) and remained approximately the same proportion to the mean thresholded acuity outwards to $10^{\circ}$ eccentricity.

Conclusion The methodology seems rapid and reliable for measuring discriminatory visual function of optotype targets throughout the central $20^{\circ}$ diameter visual field.
GA Yavuz', YB Unver ${ }^{2}$, N Bekiroglu ${ }^{3}$, P Presti ${ }^{4}$ and SH Sinclair ${ }^{5,6}$

Eye (2009) 23, 2082-2089; doi:10.1038/eye.2009.177; published online 17 July 2009

Keywords: discriminated target; perimetry; visual acuity; visual field; resolution; Landolt C

Introduction

Although vision research has explored much of the richness of the human visual system, little of this knowledge and methodology has been implemented for vision measurement within the clinic. The clinical measurement of central vision almost uniformly uses a letter chart developed more than 160 years ago by Snellen. ${ }^{1}$ Although the original chart has been modified making uniform logMAR graduations of letter size between lines, and with five letters of near similar discrimination on each line, ${ }^{2}$ nevertheless, the measurement remains restricted to defining the limits of discrimination of black, high-contrast letters against a white field at a single level of illumination in the ophthalmologist's or optometrist's office. Generally, the measurement does not evaluate the limits of vision ability under stressful encounters of reduced, colour contrasts in environments of lowered illumination or glare illumination that are frequent in daily activities. Furthermore, visual acuity represents measurement of $<1^{\circ}$ of the central field that may be at fixation or off-axis that may vary depending on the momentary fixation locus amidst pathology within the macula. The discrimination of fine letters requires fixation of the targets for prolonged periods, approaching a second or more, that is 
far removed from the usual fixations that occur in environmental scanning situations or when reading (often 200-250 ms fixations with 3-5 fixations per second $\left.{ }^{3}\right)$. Furthermore, patients vary extremely in the speed with which they read the letters on a chart, but are given the same result if the same numbers of letters are identified regardless of the speed. Often patients read a few of the letters on several lines, which may result in a variety of acuity grades among various examiners. Finally, visual acuity, as a measurement of visual performance in activities of daily living (ADL), in many studies correlates only poorly with both distance or near vision tasks. ${ }^{4-9}$

Contrast sensitivity of the central vision in the clinic has also been measured with chart systems, one using sinusoidal gratings of varying spatial frequency and contrast within spots that subtend $4^{\circ}$ of central vision, ${ }^{10}$ and one that evaluates the discrimination of letters at progressively diminished contrast. ${ }^{11}$ Although these have measured vision loss in patient's eyes wherein visual acuity testing at high contrast shows no abnormality, ${ }^{12,13}$ this chart testing, however, suffers many of the same deficiencies as the testing of high-contrast acuity since measurements are made only at the centre of the momentary fixation and at one level of illumination (although Newacheck et $a l^{14}$ has suggested a method interpreting the changes in central low-contrast acuity with alterations in luminance by using simple lowcontrast disc detection method).

Static field perimetry was originally designed to provide a method for the earlier detection of peripheral vision deficits in glaucoma or neurological disease, testing the detection of spot contrast against a diffuse, dim background at one illumination. Most often, the test is conducted without the evaluation of colour contrast perception, except for the singular short-wavelength automated perimetry that has shown earlier detection of abnormalities in glaucoma. ${ }^{15-17}$ However, contrast perimetry, when used to measure vision loss in the central field (for example, central test pattern 10-2), similar to acuity, correlates poorly with visual performance in ADL. ${ }^{18}$ Microperimetry testing integrates fundus imaging with contrast perimetry to allow the testing locations to be compared with fundus pathology, ${ }^{19-22}$ but similar to Humphrey contrast perimetry, microperimetry measures only the detection of targets contrasted against a uniformly luminant background. Discriminated targets are not evaluated, and testing cannot be performed with a variance of background luminance or hue.

Acuity perimetry was developed to measure resolution acuity at selected locations in the visual field eccentric to fixation. A prototype instrument used laser interferometry to generate a grating target at locations peripheral to a fixation target. ${ }^{23,24}$ This acuity perimeter, as well as the Frisen ring perimeter that followed, ${ }^{25}$ measured the ability to detect targets with minimum discrimination and, similar to previous perimeters, measured detection only against one level of background luminance. The group of Anderson, Beirne, Zlatkov, Demirel, and others have used equiluminant achromatic and short-wavelength opponent gratings to measure detection and resolution thresholds against a limited range of background contrast at fixation and number of limited locations to $35^{\circ}$ eccentricity. ${ }^{26-29}$ They observed that achromatic acuities were always higher than short-wavelength, colour-opponent acuities at similar eccentricities, and that at all eccentricities detection of gratings was always higher than the resolution. They have suggested that colour-opponent resolution perimetry may offer a clinical test of ganglion cell density. Using Landolt Cs, Ikeda et al ${ }^{30}$ investigated the alterations of low-contrast acuity with eccentric fixation (to a limited degree), and concluded in normal individuals that the visual acuity in the vertical and horizontal directions was slightly higher than those in the oblique directions.

An acuity perimeter has been developed (Field Acuity Analyzer, Vimetrics, LLC, Media, PA, USA) that evaluates the resolution/discrimination capabilities of vision in the central $20^{\circ}$ visual field under varying conditions of target hue, contrast, and background illumination. The device was developed to provide clinical measurement of visual performance of patients with macular disease under conditions that simulate standard conditions encountered in normal tasks of daily living. Although the potential for the instrument exists to present a myriad of hue contrasts against varying background illumination, the early testing has been conducted with black and white targets against the opponent background. This report presents the experience of testing eyes of normal individuals using high-contrast white targets presented against a black background.

\section{Materials and methods}

\section{Perimetry method}

In this prototype perimetry system, Landolt C optotypes were presented on an LCD 17-inch monitor $(1280 \times 1024$ screen resolution, Princeton Graphics, Princeton, Illinois.) placed at a distance of $86 \mathrm{~cm}$ from the participant's eye (Figure 1).

At this distance the monitor subtends a visual angle of $18^{\circ}$ vertically and $22^{\circ}$ horizontally. The participant was seated in front of the monitor with his head stabilised in a chin rest and forehead strap that could be adjusted to the 


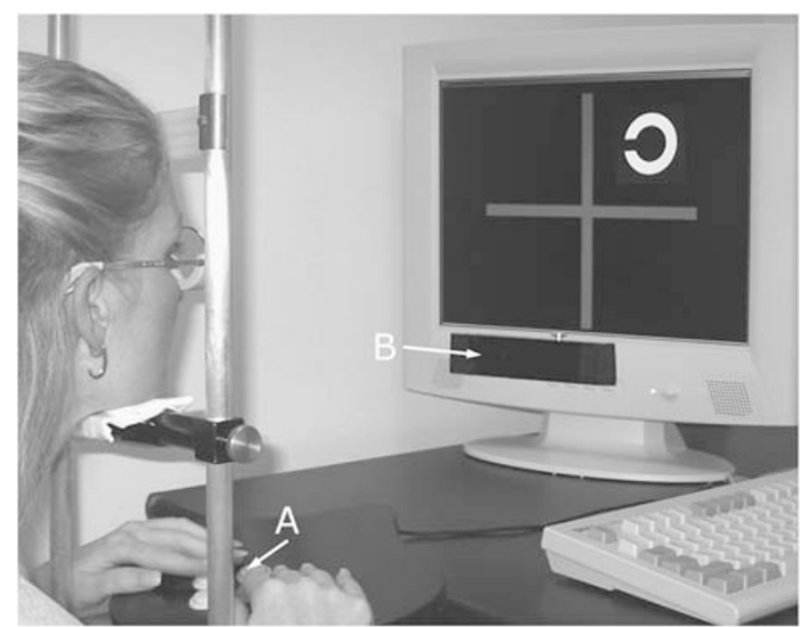

Figure 1 Testing apparatus: Patient in chin rest is tested monocularly with appropriate correction and add for screen distance. The patient signals their response to orientation of a tumbled Landolt $C$ that is flashed on screen by pressing one of the four buttons on the button pad (A). Fixation is monitored with an infrared pupil and corneal reflex camera (B) mounted in box on the bezel of monitor. The testing is conducted in a room darkened to $<1 \mathrm{Cd} / \mathrm{M}^{2}$.

height. The participant was given their full optical correction for the monitor distance. One eye is tested, whereas the other is occluded with a dark occluder. Participants were instructed to fixate a central, red, fixation cross target (subtended $15^{\circ}$ ) throughout the test. High-contrast discrimination was tested at fixation and at locations spaced throughout the visual field up to $10^{\circ}$ eccentricity. The visual discriminatory ability was determined by the participant's ability to recognise the correct orientation of a white, Landolt $\mathrm{C}$ presented for 250-ms duration with the centroid at each field location and randomly tumbled in one of the four cardinal orientations (facing up, down, right, and left). The participant's responses were recorded from a hand-held pad that contained four buttons placed in a diamond with which they indicated the direction of the opening of the Landolt $C$ after each presentation. The test was conducted in a room darkened to $<1 \mathrm{Cd} / \mathrm{M}^{2}$, with the optotype representing a $99 \%$ Michelson contrast ratio (Monaco-optix, Monaco Systems, Inc., Andover, MA, USA) against a pedestal and background of $1 \mathrm{Cd} / \mathrm{M}^{2}$ on the monitor. The pedestal obliterated the cross to a distance of 2-4 times the size of the letter. Eye fixation was monitored throughout the test (to within $0.5^{\circ}$ ) by the use of an infrared illumination and camera system with measurements at $30 \mathrm{~Hz}$ (I-Scan, Cambridge, MA, USA Figure 1); if fixation was observed to deviate during each presentation by more than $1^{\circ}$, the response was discarded and the location later retested.
The initial Landolt $C$ was presented at fixation with an optotype size of $25 \mathrm{arc}$ mins $(20 / 100)$ and was then reduced or enlarged at the next presentation depending on whether a correct or incorrect response was elicited. Large steps (of enlargement or reduction in letter size) were used until the first reversal response, after which progressively smaller steps were used to step back and forth to define the threshold; the threshold was defined as the smallest size letter for which a correct response was elicited with two incorrect responses for the next optotype size smaller. The finest steps of optotype size were $0.1 \log$ MAR (similar to the ETDRS chart in which three steps equal a doubling of the subtended visual angle). The smallest $C$ size that could be presented, given the pixel density of the monitor and the participant's distance, subtended $5 \mathrm{~min}$ of arc $(20 / 20)$, and the largest $80 \mathrm{~min}(20 / 320)$. After thresholding for the smallest optotype discriminated at fixation, the Landolt Cs were presented and thresholded with the same techniques at each of the peripheral locations presenting randomly among the locations, such that the person could not predict where on the monitor the next presentation would appear. Intervals between presentations were adjusted to the person's responses, ranging from 1.0 to $4.0 \mathrm{~s}$.

\section{Participants}

Volunteers of varying age were solicited among family members of patients in a retina practice. The participants selected had never before undergone the testing, and were included in the study if both eyes were determined to be normal through evaluation of visual acuity, IOP measurement, and slit lamp examination of the anterior segment and of the optic nerve and macula. The bestcorrected visual acuity (ETDRS chart, Lighthouse, New York City, NY, USA) was measured, and was required to be $20 / 30$ or better with a spherical refractive error of $< \pm 4.00 \mathrm{D}$ and with an astigmatic correction of $<2.00 \mathrm{D}$. The intraocular pressure was required to be $\leqslant 21 \mathrm{~mm} \mathrm{Hg}$, and the lens opacity for older individuals, graded by the LOCS III system, ${ }^{31}$ no greater than NO2, NC2, $P<1, C<1$. Participants were excluded if they had a medical history of diabetes, hypertension, or other systemic or neurological disease that would affect vision, or if there was a history of retinal or optic nerve disease, ocular trauma, or surgery. All patients who were enrolled in the study gave informed consent, and all individuals who participated in this study are included in this report. The research adhered to the tenets of the Declaration of Helsinki.

Testing with the discriminated target perimetry system was conducted on the same day as the chart testing and examination of the eye. The eye that was tested and 
included in the study was chosen at random. Twentyfour of the participants underwent repeated perimetry testing after 1-30 days to evaluate for a learning curve and for test-retest repeatability for the acuity thresholded at each eccentricity.

\section{Statistics}

To define the mean values at each eccentricity, the thresholded acuity values obtained at all locations (in minutes of arc, minimum angle of resolution (MAR)) for a given eccentricity were averaged for each eye and then the means averaged for all eyes were measured. Learning was evaluated by subtracting the value obtained at each location in the second test from that obtained in the first and then averaging the differences for similar eccentricities for each eye and then averaging among all eyes. If there were a significant learning experience, this should result in an overall improvement in the resolution at each location in the second test relative to that of the first test result and a positive difference. If there is no or minimal learning effect (the mean of the differences between the first and second test at each eccentricity

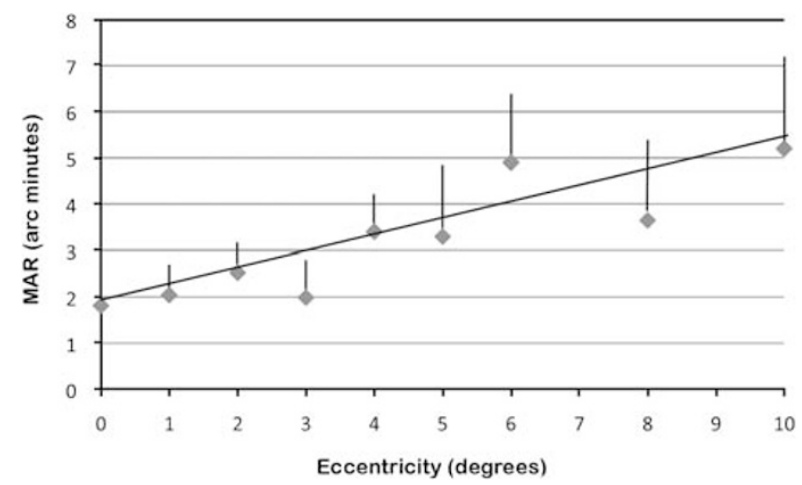

Graph 1 Minimum angle of resolution (the angle subtended by the Landolt $\mathrm{C}$ gap) plotted against eccentricity (in degrees) from fixation. Linear regression results are plotted as a line. Vertical bars represent $+1 \mathrm{SD}$ from the mean. approach 0 ), the test-retest repeatability may be determined at each eccentricity by calculating the SD of the differences averaged for the eyes undergoing the test-retest evaluation. The coefficient of repeatability, representing $95 \%$ confidence limits for a normally distributed population of eyes, ${ }^{32}$ was calculated at fixation and at each eccentricity tested.

\section{Results}

Seventy individuals, 33 men and 37 women, underwent testing of one eye, chosen at random. Ages ranged from 18 to 77 years with a mean of $39.5 \pm 14.7$ ( \pm 1 SD) years. The duration of perimetry testing averaged 8-10 min.

The mean and SD of the high-contrast acuities, evaluated as a function of eccentricity from fixation, are plotted in Graph 1. The mean ETDRS acuity measured $1.15 \pm 0.37$ arc min MAR $(20 / 23)$, whereas the acuity thresholded at fixation determined by the field-testing method measured $1.75 \pm 0.85$ arc min MAR (20/35).

The discriminated acuity declined with increasing eccentricity, in what appeared to be a linear fashion, to $3.42 \pm 2.25 \mathrm{arc} \min$ at $5^{\circ}(20 / 68$ equivalent $)$ and $5.81 \pm 3.97$ arc $\min (20 / 116$ equivalent $)$ at $10^{\circ}$ eccentricity. Linear regression analysis between visual acuity and eccentricity was Vmar $=1.74+0.330$ (eccentricity in degrees) $\left(R^{2}=0.966, P<0.0001\right)$. The results are presented in Graph 1 .

The analysis of repeated testing to determine the effects of learning showed minimal learning effects (5-8\% of the average thresholded optotype size) at all eccentricities up to $8^{\circ}$. At $8-10^{\circ}$ from fixation, a slightly greater learning effect was noted, $16-25 \%$ of the average thresholded optotype size, but the effect was still $<0.1 \log$ MAR. The coefficient of test-retest repeatability, ${ }^{33}$ calculated as 1.96 times one SD of the differences and representing 95\% confidence limits, at fixation measured 1.12 MAR, which represented $64 \%$ of the mean acuity thresholded at fixation. This relationship

Table 1 Coefficients of repeatability for 24 eyes that underwent repeat testing

\begin{tabular}{lcccc}
\hline Eccentricity degrees & $\begin{array}{c}\text { Mean MAR } \\
\text { of 1st test }\end{array}$ & $\begin{array}{c}\text { SD of difference } \\
\text { of mean MAR }\end{array}$ & $\begin{array}{c}\text { Coefficient of } \\
\text { repeatability (CR) }\end{array}$ & $\begin{array}{c}\text { CR as a \% of } \\
\text { mean MAR }\end{array}$ \\
\hline 0 & 1.81 & 0.56 & 1.12 & $62 \%$ \\
1 & 2.04 & 0.61 & 1.19 & $59 \%$ \\
2 & 2.52 & 0.61 & 1.20 & $48 \%$ \\
3 & 1.99 & 0.63 & 1.24 & $63 \%$ \\
4 & 3.39 & 0.97 & 1.91 & $56 \%$ \\
5 & 3.29 & 1.32 & 2.60 & $30 \%$ \\
6 & 5.24 & 0.93 & 3.00 & $35 \%$ \\
8 & 3.64 & 1.53 & 3.80 & $77 \%$ \\
10 & 4.96 & 1.94 & & $73 \%$ \\
\hline
\end{tabular}

MAR, minimum angle of resolution; SD, standard deviation.

${ }^{\mathrm{a}} \mathrm{CR}=1.96 \times \mathrm{SD}$ of differences of mean MAR (95\% confidence limits). 
of test-retest repeatability, measured at each location, remained at a similar proportion of the thresholded acuity at all eccentricities tested (please see Table 1).

\section{Discussion}

This study describes an automated method to measure thresholds of visual discrimination for discriminated targets within the central $10^{\circ}$ radius visual field. Although the system allows the versatility to test discrimination under other levels of contrast, illumination, and hue, in this study, normal values are reported only for high-contrast white targets presented against a black screen in a darkened room $(99 \%$ Michelson contrast, background $1.0 \mathrm{Cd} / \mathrm{M}^{2}$ ).

Visual acuity, as determined with the ETDRS chart, averaged $1.15 \pm 0.37$ arc min MAR (20/23), which compares with prior studies using the same chart, ${ }^{32}$ whereas the acuity thresholded at fixation by the perimetry system averaged $1.75 \pm 0.85$ arc min MAR (20/35) representing 0.6 arc min MAR poorer than the ETDRS acuity. This difference may have been due to the short presentation time of the Landolt Cs ( $250 \mathrm{~ms})$ compared with the average duration of fixation that is usually taken to read each letter of the acuity chart (usually to $0.5-1 \mathrm{~s}$ or more). ${ }^{34}$ Discrimination of targets at fixation has been shown in prior studies to require longer presentation times than that required for detection, ${ }^{34}$ but has been observed to be independent of the presentation time in normal eyes for times longer than $180 \mathrm{~ms} .{ }^{35}$ The relatively larger acuity, determined by the Landolt $\mathrm{C}$ thresholding method, in this study may also have been due to the methodology for thresholding or to the use of white letters against a black background, which in a darkened room would result in relative pupil dilation and poorer resolution. However, this may have been offset by the reduced glare compared with a black letter presented against a white background. ${ }^{36}$ Finally, the larger mean value reported by the thresholding technique at fixation may also have been caused by a truncation of better resolutions, caused by the inability of the visual field monitor to produce Landolt Cs with an opening smaller than 1 arc min $(20 / 20)$.

The MAR of the discriminated targets deteriorated to $3.42 \pm 2.25$ arc $\min$ MAR $(20 / 68)$ at $5^{\circ}$ eccentricity and to $5.81 \pm 3.97$ arc $\min (20 / 116)$ at $10^{\circ}$ eccentricity (Graph 1$)$. These results, obtained on naïve individuals, are similar to that determined in prior investigations of laboratory investigators using sinusoidal gratings, ${ }^{34,35}$ Landolt C rings, ${ }^{37-40}$ and Snellen letters ${ }^{41-43}$ with similar presentation times. Similar to most authors, we also showed a linear deterioration of acuity with eccentricity. Some authors ${ }^{40}$ have found a minor break between $0.5^{\circ}$ and $1^{\circ}$; however, because of the spacing of locations within the visual field in this study, a break from linearity could not be detected. Comparing nasal vs temporal presentations, or superior vs inferior, we observed no significant differences at any eccentricity with a two-tailed, paired $t$-test at $P<0.05$, results similar to that of prior studies. ${ }^{32,39,44-46}$

Refixations may potentially produce artifacts in the measurement of the acuities at the locations close to fixation. However, for an eccentric stimulus to initiate a saccade in the appropriate direction, it must persist for at least a minimum time of 100-120 ms for detection, ${ }^{47}$ after which the saccade would require $\sim 20-35 \mathrm{~ms}$ to complete the refixation over the angular distance, and thereafter, the target must remain for at least 150-180 m for discriminatory function to ascertain the correct $\mathrm{C}$ orientation. As the target is presented for a duration of only $250 \mathrm{~ms}$, and randomly throughout the visual field, the possibility that refixation may account for the values obtained at eccentric loci is eliminated. In addition, as the fixation is measured at $30 \mathrm{~Hz}$ with a $0.5^{\circ}$ accuracy, and those presentations in which refixations did occur $\left(>1^{\circ}\right.$ deviation from primary fixation at the completion of the peripheral element presentation) were eliminated, artifacts caused by refixation, we believe, are essentially prevented.

Chart acuity or contrast sensitivity has been observed to correlate poorly with visual function during $\mathrm{ADL}^{48}$ perhaps because the fixations that are commonly used during these activities (that is, $234 \mathrm{~ms}$ during reading, $200 \mathrm{~ms}$ while driving, or $220 \mathrm{~ms}$ for still-image viewing ${ }^{47,48}$ ) are far shorter than the fixation times used during chart reading, often reaching $1 \mathrm{~s}$ or more, and often longer for a person with macular disease. The presentation time of $250 \mathrm{~ms}$ that was used in this study for $\mathrm{C}$ presentation was chosen because it closely approximates the fixation times required for a number of tasks in ADL, and for this reason, we believe the field acuities measured by this means should correlate better with the vision that is required for ADL than acuities obtained from reading charts. However, this relationship will necessarily have to be tested in future trials.

There are a number of methods that have been proposed for approaching a threshold during visual psychophysical testing. King-Smith et al have developed the ZEST method with QUEST evaluations, ${ }^{49,50}$ whereas Anderson et al have proposed another method (used in the Humphrey matrix perimeter) that makes use of the information gained from every response at a given location to determine the subsequent and final estimates. ${ }^{51}$ The thresholding methodology for determining the acuity at a particular field location that was used in the instrument described pursued progressively finer logMAR optotype reversals across 
the purported threshold with methods to correct for mistakes. This methodology represents an alternative that borrows from the above methods and, we believe, represents a clinically reasonable and succinct method for approaching the threshold. Measurement of a psychophysical threshold, which ideally would use a probit analysis with approach from both the supra as well as infra-response positions, is dependent on the number of inquiries as well as the size of steps of those inquiries. $^{52}$ The ETDRS chart testing, which uses a $1: 12$ chance of guessing correctly among the Sloan letter set, has been shown to produce high reproducibility but to have poor sensitivity because the steps between the lines (0.1 logMAR) have been shown to be larger than optimal. ${ }^{53}$ In addition, during testing with charts in the clinic, often the approach to threshold is truncated because the patient is rarely and variably encouraged to continue to progress downward and to 'guess' until a complete line of five letters is missed (as per the recommendations of the ETDRS manual). Using a single positive end point with the requirement of two incorrect responses at the next lower optotype size in the Landolt $\mathrm{C}$ thresholding means that the threshold, with a one-infour chance of guessing, would have a $14 \%$ chance of producing a threshold one step (0.1 logMAR) lower than the true threshold, and a $3.5 \%$ chance of reporting a threshold two steps lower. We are currently pursuing evaluation of a system that would utilise a required double-correct response at the lowest optotype step with two incorrect responses at the next step smaller and, in addition, would use $0.05 \log$ MAR steps as recommended by Wood and Bullimore ${ }^{53}$ and Arditi et al, ${ }^{54}$ in order to achieve greater sensitivity.

With the current visual field instrument and $0.1 \log$ MAR steps, in this study of 24 of the individuals, a test-retest reliability of 1.12 arc min MAR (95\% confidence limits) was achieved at fixation, appearing better than studies of experienced and motivated participant that measured the ETDRS chart acuity with line-by-line scoring (95\% confidence limits 1.8 MAR), and similar to that using letter-by-letter scoring (95\% confidence limits, eight letters). ${ }^{54}$ Results of retesting showed minimal learning except at the most peripheral eccentricities (16-25\% improvement of the thresholded value at each eccentricity).

In addition to the high-contrast white-on-black testing performed in the study reported here, the technology and methods enable the optotype as well as pedestal and background contrast, luminance, and hue to be varied, in order to explore the central field of vision for an individual in multiple dimensions. The results of such a test provide the opportunity for a much more complete analysis of the visual capacity in different environmental conditions, including those that stress the visual abilities.
Such a 'stress test' of vision offers the potential for improved monitoring and earlier detection of ocular pathology than that available with chart testing or with contrast-only field testing. We recognise that individuals with macular pathology and central or paracentral scotomas may shift fixation loci between episodes of testing, causing variability of the results at individual loci. ${ }^{55}$ However, measures of more global macular function may be derived from the visual field test results. For instance, around any particular fixation locus, an average may be calculated from the acuities obtained at all locations that is weighted inversely with distance from fixation that would provide an overall measure of integrated macular function. One could also determine the area and form factor of the visual field at any desired level of spatial sensitivity (that is, the 'hill of vision' could be sectioned to determine the total area and form at any desired level of acuity), although there possibly may be variable depressions or scotomas within that field.

Finally, for particular tasks such as reading that can be severely limited by paracentral scotomas, one may wish to know the position and size within the field of the horizontal window that would maximise the number of letters that could be viewed simultaneously (that is, a horizontal 'instantaneous field of view'). We believe that such measures will provide a far more rich understanding of the abilities and shortcomings of vision in individuals with macular or optic nerve pathology (as well as the effect of media haze that reduces contrast) that will empower the clinician to provide more effective care and more appropriate vision rehabilitation.

\section{References}

1 Bennett A. Ophthalmic test types. Br J Physiol Opt 1965; 22: 236-271.

2 Ferris FL, Kassoff A, Breswick GH, Bailey I. New visual acuity charts for clinical research. Am J Ophthalmol 1982; 94(1): 91-96.

3 Fischer B, Ramsberger E. Human express saccades: extremely short reaction times of goal directed eye movements. Experimental Brain Research 1984; 57: 191-195.

4 Ball K, Owsley C. The useful field of view test: a new technique for evaluating age-related declines in visual function. J Am Optom Assoc 1992; 63: 71-79.

5 Goodrich G. A picture without meaning isn't worth a thousand words: visual information in vision rehabilitation. Low Vision. IOS press: 1994 pp 111-120.

6 Legge G, Ross J, Isenberg L, LaMay J. Psychophysics of reading: clinical predictors of low-vision reading speed. Invest Ophthalmol Vis Sci 1992; 33(3): 677-687.

7 Dodds A, Davis D. Assessment and training of low vision clients for mobility. J Vis Impair Blind 1989; 70: 439-446.

8 Owsley C, Ball K, McGwin G, Sloane ME, Roenker DL, White MF et al. Visual processing impairment and risk of 
motor vehicle crash among older adults. JAMA 1998; 279: 1083-1088

9 Owsley C, Sloane Jr GM, Stalrey BT, Wells J. Timed instrumental activities of daily living tasks: relationship to visual function in older adults. Optom Vis Sci 2001; 78(5): 350-359.

10 Ginsberg A. A new contrast sensitivity vision test chart. Am J Optom Physiol Opt 1984; 61: 403-407.

11 Pelli D, Robson J, Wilkins A. The design of a new letter chart for measuring contrast sensitivity. Clin Vision Sci 1988; 2: 187-199.

12 Regan D, Neima D. Low-contrast letter charts as a test of visual function. Ophthalmology 1983; 90: 1192-1200.

13 Regan D, Neima D. Low-contrast letter charts in early diabetic retinopathy, ocular hypertension, glaucoma, and Parkinson's disease. Br J Ophthalmol 1984; 68: 885-889.

14 Newacheck JS, Haegerstrom-Portnoy G, Adams AJ. Predicting visual acuity from detection thresholds. Optom Vis Sci 1990; 67: 184-191.

15 Johnson C, Brandt J, Khong AM, Adams A. Shortwavelength automated perimetry in low, medium and high-risk ocular hypertensive eyes. Arch Ophthalmol 1995; 113: 70-76.

16 Harwerth RS, Smith 3rd EL, DeSantis L. Mechanisms mediating visual detection in static perimetry. Invest Ophthalmol Vis Sci 1993; 34(10): 3011-3023.

17 Johnson CA, Adams AJ, Casson EJ, Brandt DM. Blue on yellow perimetry can predict the development of glaucomatous field loss. Arch Ophthalmol 1991; 111: 645-650.

18 Nelson P, Aspinall P, O'Brien C. Patients perception of visual impairment in glaucoma: a pilot study. $\mathrm{Br} \mathrm{J}$ Ophthalmol 1999; 83(5): 546-552.

19 Fujii GY, De Juan Jr E, Humayun MS, Sunness JS, Chang TS, Rossi JV et al. Characteristic of visual loss by scanning laser ophthalmoscope microperimetry in eyes with subfoveal choroidal neovascularization secondary to age-related macular degeneration. Am J Ophthalmol 2003; 136(6): 1067-1078.

20 Charbel IP, Helb HM, Rohrschneider K, Holz FG, Scholl HP. Microperimetric assessment of patients with type 2 idiopathic macular telengiectasia. Invest Ophthalmol Vis Sci 2007; 48(8): 3788-3795.

21 Jackson GR, Owsley C. Visual dysfunction, neurodegenerative disease and aging. Neurol Clin 2003; 21(3): 709-728.

22 Midena E. Perimetry and the Fundus: an Introduction to Microperimetry, 1st edn. Slack Inc Thorofare, NJ, 2006, pp 15-26.

23 Phelps CD. Acuity perimetry and glaucoma. Trans Am Ophthalmol Soc 1984; 82: 753-791.

24 Thibos L. Acuity perimetry and the sampling theory of visual resolution. Optom Vis Sci 1998; 75(6): 399-406.

25 Frisen L. Acuity perimetry: estimation of neural channels. Int Ophthalmol 1988; 12(3): 169-174.

26 Anderson RS, Coulter E, Zlatkova MB, Demirel S. Short wavelength acuity: optical factors affecting detection and resolution of blue-yellow sinusoidal gratings in foveal and peripheral vision. Vision Res 2000; 43(1): 101-107.

27 Beirne RO, Zlatkova MB, Anderson RS. Changes in human short-wavelength-sensitive and achromatic resolution acuity with retinal eccentricity and meridian. Vis Neurosci 2005; 22(1): 79-86.
28 Beirne RO, Zlatkova MB, Chang CK, Chakravarthy U, Anderson RS. How does the short-wavelength-sensitive contrast sensitivity function for detection and resolution change with age in the periphery? Vis Res 2008; 48(18): 1894-1901.

29 Demirel S, Anderson RS, Haggerty K, Thibos LN. Detection and resolution of high-pass (vanishing-optotype) letters in central and peripheral vision. Optom Vis Sci 1998; 75: 202.

30 Ikeda K, Noda K, Yamaguchi S. Visual acuity as a function of luminance contrast of Landolt rings and adaptation luminance. J Light Vis Env 1983; 7: 28-34.

31 Chylack Jr LT, Wolfe JK, Singer DM, Leske MC, Bullimore MA, Bailey IL et al. The Lens Opacities Classification III System. The Longitudinal Study of Cataract Study Group. Arch Ophthalmol 1993; 111(6): 831-836.

32 Rosser DA, Murdoch IE, Fitzke FW, Laidlaw DA. Improving on ETDRS acuities: design and results for a computerized thresholding device. Eye 2003; 17(6): 701-706.

33 Bland JM, Altman DG. Statistical methods for assessing agreement between two methods of clinical measurement. Lancet 1986; 1(8476): 307-310.

34 Graham C, Cook C. Visual acuity as a function of intensity and exposure time. Am J Psychol 1937; 49: 654-661.

35 Wertheim T. Uber die indirekte sehscharfe. Zft $f$ Sinnesphysiol 1894; 7: 172-189.

36 González EG, Tarita-Nistor L, Markowitz SN, Steinbach MJ. Computer-based test to measure optimal visual acuity in age-related macular degeneration. Invest Ophthalmol Vis Sci 2007; 48(10): 4838-4845.

37 Weymouth F, Hines D, Acres L, Raaf JE, Wheeler MC. Visual acuity within the area centralis and its relation to eye movements and fixation. Am J Ophthal 1928; 11: 947-960.

38 Fick A. Uber Stabchensehscharfe und Zapfensehscharfe. Arch of Ophthal 1898; 45: 336-356.

39 Ruppert L. Ein vergleich zwischen dem Distinktionsvermogen und der Bewegungsempfindlichkeit der Netzhautperipherie. Zeitschrift fur Sinnesphysiologie 1908; 42: 409-423.

40 Mandelbaum J, Sloan L. Peripheral visual acuity. Am J Ophthal 1947; 30: 581-588.

41 Millodot M. Foveal and extra-foveal acuity with and without stabilized retinal images. Br J Physiol Opt 1966; 23(2): 75-106.

42 LeGrand Y. Optique physiologique. 3rd Vol L'espace Visuel. Revue d'Optique: Paris, 1956 pp 391-392.

43 Bailey FL, Bullimore MA, Raasch TW, Taylor HR. Clinical grading and the effects of scaling. Invest Ophthalmol Vis Sci 1991; 32: 422-432.

44 National Research Council Committee on Vision. Recommended standards for the clinical measurement and specification of visual acuity. Adv Ophthalmol 1980; 40: 1.

45 Jonkers GH, Burger J. The determination of the central static visual acuity, presentation of a modified Landolt $\mathrm{C}$ chart. Doc Ophthalmol 1991; 78(3-4): 219-223.

46 Ruamviboonsuk P. Repeatability of an automated Landolt C test, compared with the ETDRS chart testing. Am J Ophthalmol 2003; 136(4): 662-669.

47 Yarbus AL. Eye Movements and Vision. Plenum Press: New York, 1967, pp 1-222.

48 Laitinen A, Sainio P, Koskinen S, Rudanko SL, Laatikainen $\mathrm{L}$, Aromaa A. The association between visual acuity and functional limitations: findings from a nationally 
representative population survey. Ophthalmic Epidemiol 2007; 14(6): 333-342.

49 King-Smith PE, Grigsby SS, Vingrys AJ, Benes SC, Supowit A. Efficient and unbiased modifications of the QUEST threshold method: theory, simulations, experimental evaluation and practical implementation. Vision Res 1994; 34(7): 885-912.

50 King-Smith PE, Rose D. Principles of an adaptive method for measuring the slope of the psychometric function. Vision Res 1997; 37(12): 1595-1604.

51 Anderson AJ, Johnson JA, Fingeret M, Keltner JL, Spry PG, Wall $\mathrm{M}$ et al. Characteristics of the Normative Database for the Humphrey matrix perimeter. Invest Ophthalmol Vis $\mathrm{Sci}$ 2005; 46: 1540-1548.

52 Finney DJ, Stevens WL. A table for the calculation of working probits and weights in probit analysis. Biometrika 1948; 35(pts 1-2): 191-201.

53 Wood JM, Bullimore MA. Changes in the lower displacement for motion with age. Ophthalmic Physiol Opt 1995; 15(1): 31-36.

54 Arditi A. Improving the design of the letter contrast sensitivity test. Invest Ophthalmol Vis Sci 2005; 46: 2225-2229.

55 Bullimore MA, Bailey IL. Reading and eye movements in age-related maculopathy. Optom Vis Sci 1995; 72(2): 125-138. 